\title{
Chronic Disease Prevention Particularly Focus on Obesity
}

\author{
Eldoom EA ${ }^{*}$, Mohammed AHA ${ }^{2}$ and Ahmed AAA $^{3}$ \\ ${ }^{1}$ Faculty of Public Health University of Alzaeim Alazhari/ Sudan \\ 2UNFPA Nyala office \\ ${ }^{3}$ University of Nyala- Faculty of Medicine registrar
}

Research Article

Volume 3 Issue 2

Received Date: June 04, 2018

Published Date: June 15, 2018

*Correponding author: Ekram Adam Eldoom Faculty of Public Health University of Alzaeim Alazhari/ Sudan, Tel: 00249912103557; Email: ekram.eldoom3@gmail.com

\section{Abstract}

The study was describe the relationship between obesity (over weight and prevalence of health conditions and chronic disease (CAD, diabetic, hypertension) in Nyala university staff, a total of (50participants) over 25 years old and above were involved, a pretested self-administered questionnaire was used for data collection. Results revealed that, $45 \%$ of overweight had hypertension, 36\% had DM, $9 \%$ had CVD \& 9\% had obesity. The prevalence of obesity-related co morbidities emphasizes the need for concerted efforts to prevent and treat obesity rather than just its associated co morbidities. The study recommends that the health care system must increasingly be overwhelmed with individuals who require treatment for obesity-related health conditions.

Keywords: Obesity; Prevalence; Hypertension; Overweight; Co morbidities

\section{Introduction}

Overweight and obesity are defined as abnormal or excessive fat accumulation that presents a risk to health. A crude population measure of obesity is the body mass index (BMI), a person's weight (in kilograms) divided by the square of his or her height (in metres). A person with a BMI of 30 or more is generally considered obese. A person with a BMI equal to or more than 25 is considered overweight.

Overweight and obesity are major risk factors for a number of chronic diseases, including diabetes, cardiovascular diseases and cancer. Once considered a problem only in high income countries, overweight and obesity are now dramatically on the rise in low- and middle-income countries, particularly in urban settings [1].

\section{Obesity Affects Some Groups More than Others}

Non-Hispanic blacks have the highest age- adjusted rates of obesity $(48.1 \%)$ followed by Hispanics (42.5\%), non-Hispanic whites (34.5\%), and non-Hispanic Asians $(11.7 \%)$. Obesity is higher among middle age adults age $40-59$ years $(40.2 \%)$ and older adults age 60 and over (37.0\%) than among younger adults age 20-39 (32.3\%). Obesity-related conditions include heart disease, stroke, type 2 diabetes and certain types of cancer, some of the leading causes of preventable death [2].

Some researchers have suggested that diets with a high energy density, referring to the amount of energy per 
volume, offer an alternative explanation for the observed increases in obesity, but long-term studies have not examined this theory. Sugar-sweetened beverages contribute significantly to the overconsumption of calories, in part because calories in fluid form appear to be poorly regulated by the body [3]. Obesity also adds to risk once the levels of coexisting risk factors are taken into account. Obesity is associated with elevated blood pressure, blood lipids, and blood glucose; changes in body weight are coincident with changes in these risk factors for disease.

\section{Epidemiological Determinants}

The etiology of obesity is complex and is one of multiple causation

a. Age: obesity can occur at any age, and generally increases with age

b. Sex: Women generally have higher rate of obesity than men, although may have higher rates of overweight.

c. Genetic factor: There is a genetic component in the etiology of obesity.

d. Physical activity: there is convincing evidence that regular physical activity is protective against unhealthy weight gain.

e. Socioeconomic status [4]

\section{Causes of Obesity}

\section{Consuming too Many Calories}

These days' people are eating much more food than in previous generations. This used to be the case just in developed nations - however, the trend has spread worldwide.

\section{Leading a Sedentary Lifestyle}

With the arrival of televisions, computers, video games, remote controls, washing machines, dish washers and other modern convenience devices, people are commonly being leading a much more sedentary lifestyle compared to their parents and grandparents.

\section{Not Sleeping Enough}

Research has suggested that if you do not sleep enough your risk of becoming obese doubles. Research was carried out at Warwick Medical School at the University of Warwick. The risk applies to both adults and children. Professor Francesco Cappuccio and team reviewed evidence in over 28,000 children and 15,000 adults. Their evidence clearly showed that sleep deprivation significantly increased obesity risk in both groups.

\section{- Professor Cappuccio said}

"The 'epidemic' of obesity is paralleled by a 'silent epidemic' of reduced sleep duration with short sleep duration linked to increased risk of obesity both in adults and in children". These trends are detectable in adults as well as in children as young as 5 years [5].

\section{Endocrine disruptors, such as some foods that interfere with lipid metabolism}

Fructose is mainly metabolized in the liver, the target organ of the metabolic alterations caused by the consumption of this sugar. The rats receiving fructosecontaining beverages presented pathology similar to metabolic syndrome, which in the short term causes lipid accumulation (hypertriglyceridemia) and fatty liver, and eventually leads to hypertension, resistance to insulin, diabetes and obesity. Poorly balanced diets and the lack of physical exercise are key factors in the increase of obesity and other metabolic diseases in modern societies. In epidemiological studies in humans, the effect of the intake of fructose-sweetened beverages also seems to be more intense in women [6].

\section{Lower Rates of Smoking (Smoking Suppresses Appetite)}

According to the National Institutes of Health (NIH) "Not everyone gains weight when they stop smoking. Among people who do, the average weight gain is between 6 and 8 pounds. Roughly 10 percent of people who stop smoking gain a large amount of weight - 30 pounds or more.

\section{Medications that Make Patients Put on Weight}

According to an article in Annals of Pharmacotherapy, some medications cause weight gain. "Clinically significant weight gain is associated with some commonly prescribed medicines. There is wide inter individual variation in response and variation of the degree of weight gain within drug classes where possible, alternative therapy should be selected, especially for individuals predisposed to overweight and obesity $[7,8]$.

\section{Obesity Self-Perpetuating}

The longer a person is overweight, the harder it becomes for them to lose weight. Many have wondered whether obesity itself becomes a permanent state, i.e. does obesity promote obesity?. Researchers from the University of Michigan and the National Council of Science and Technology (COINCET) in Argentina, reported in the Journal of Clinical Investigation that in animal experiments, obesity seems to become a self-perpetuating state. 
They found that the "normal" body weight of mice that become obese starts going up; their bodies' perception of normal weight becomes a heavier than before, regardless of whether they are made to go on diets which had made them lose weight.

\section{Obesity Gene}

A faulty gene, called FTO, makes 1 in every 6 people overeat,

\section{Methods}

Across sectional study was conducted in Nyala university staff (4 group were from 4 collage which faculty of engineering, veterinary, low, science and information technology are and one group from administrative staff) , a total of 50 Nyala university staff were involved in this study using stratified random sampling. A pretested self-administered questionnaire was used for data collection and they were asked to write answer of the question on their own. Questionnaire was including personal data, measurement of weight and height, current and past medical history of chronic disease, first degree relative medical history for chronic disease and habits and practice including exercise, smoking sedentary life style.

\section{Result}

In Figure 1 showed strong relation between the age and chronic disease.

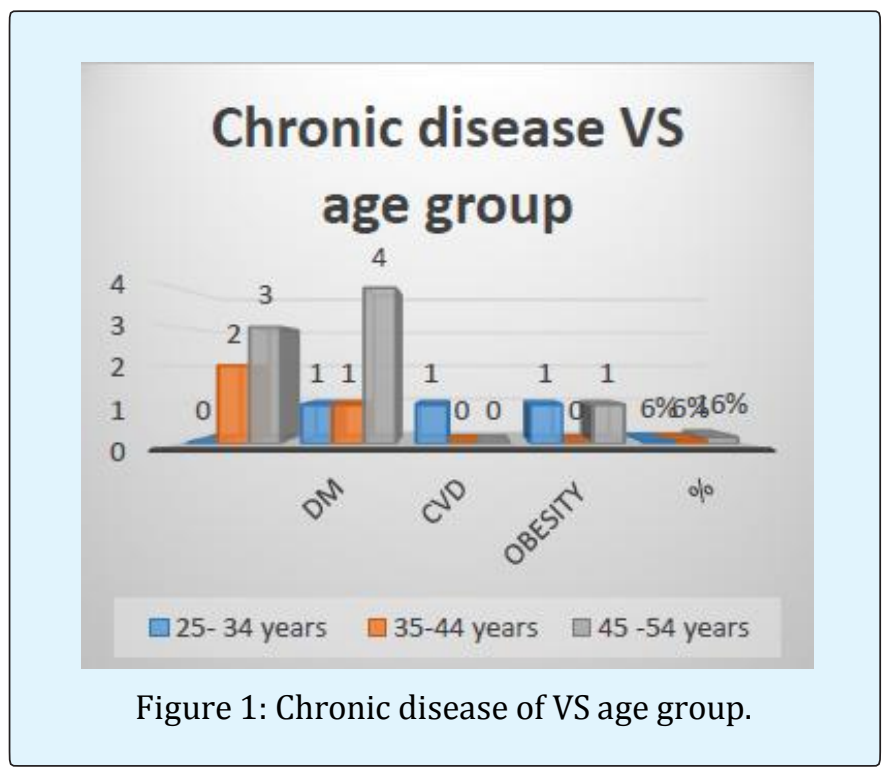

Figure 2 reveal association between chronic disease and MBI.

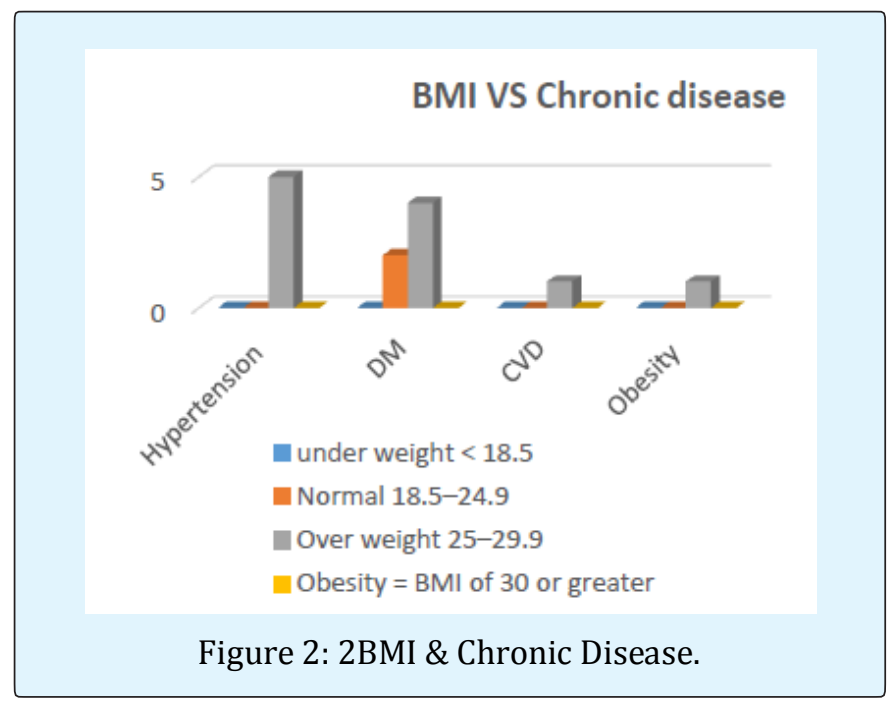

Figure 3 studied the relation between chronic diseases and over weigh

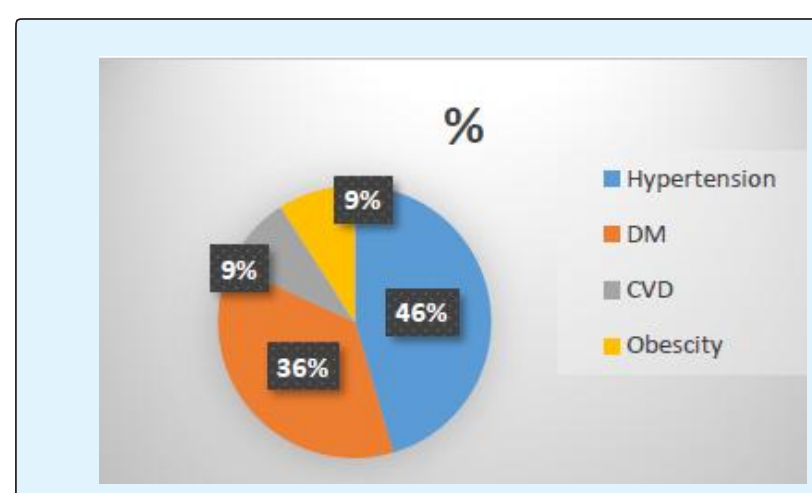

Figure 3:Chroniv disease among of over weight.

In Figure 4 find that strong association and correlation between increase in family history and chronic disease.

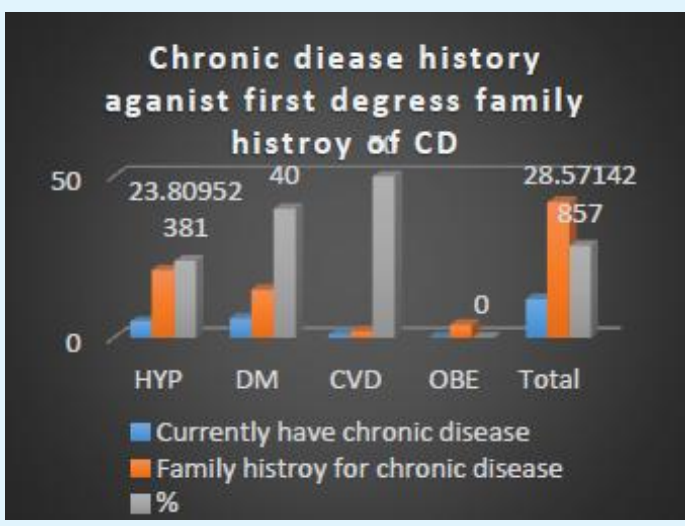

Figure 4: History(participants)of CD cs family history of CD. 
Figure 5 explained the association between habits \& chronic disease

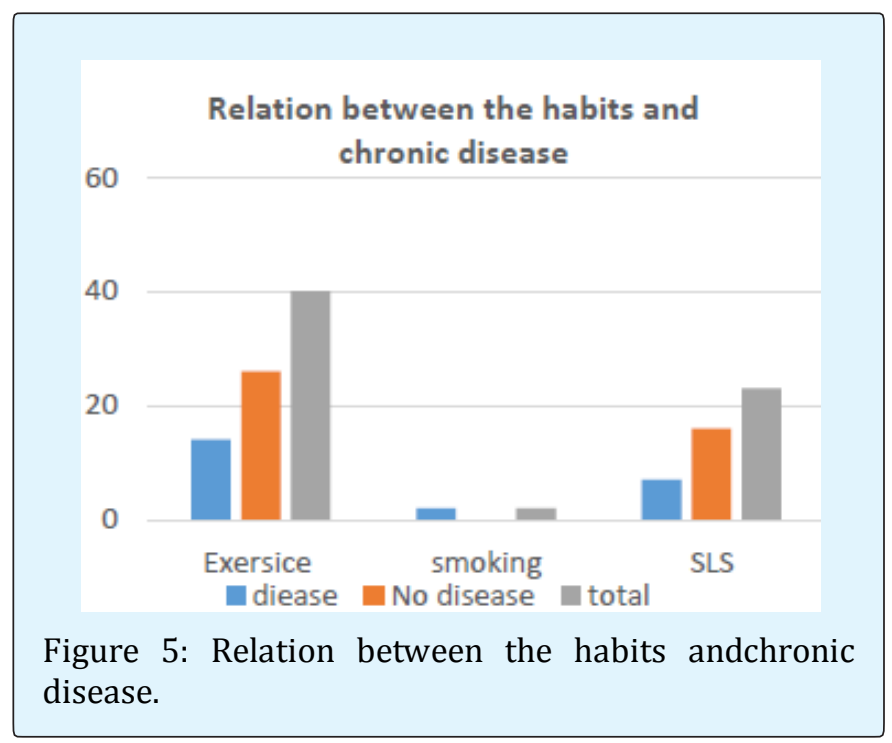

\section{Discussion}

In figure 1 showed that total of 50 participants were studied we legalized that there is strong relation between the age and chronic disease $16 \%$ of chronic disease were occur in age group between $45-54$ years old this similar as stated by [1].

As shown in figure 2 both men and women, high blood pressure was the most common overweight- and obesityrelated health condition and its prevalence showed a strong increase with increasing weight status .The prevalence of DM, increased sharply among both overweight and obese men and women corresponding with the increasing weight classes. However, men and women with BMIs of $25 \mathrm{~kg} / \mathrm{m} 2$ or more, were more likely than persons of normal weight to have high blood pressure levels this agree with mentioned by Obesity is associated with elevated blood pressure, blood lipids, and blood glucose; changes in body weight are coincident with changes in these risk factors for disease" $[3,4]$.

In this study, figure 3 estimated that relationship between overweight and obesity class levels and morbidity in a contemporary, nationally representative sample of adults. We observed a substantial prevalence of chronic health conditions in association with elevated BMI for both age groups and across racial and ethnic groups. Associations of weight status and health outcomes did not differ between the 3 major racial and ethnic groups. The PRs generally increased with increasing severity and, for many co morbidities, the PRs were significantly elevated even for the overweight class (BMI, 25.0-29.9 kg/m2). Because the data reflect that the burden of disease associated with overweight and obesity in the Nyala university staff population age between 25 54 years old this agree with [5].

We observed that the relationship between obesity class and co morbidities was generally strongest among the younger age groups. PRs were significantly elevated in the older age group except for gallbladder disease in men and high cholesterol level in both sexes this agree with [2]. Also there are relation between habits and family history as shown in figures $4 \& 5$ these agree with [4].

Our approach has several limitations. Foremost, a cross-sectional approach to evaluate the relationship of obesity to the morbidities that we examined does not provide evidence for causality. In some individuals, weight loss may accompany some of these conditions, particularly CHD. Because persons in the higher obesity classes were at increased risk of death, we may have underestimated the impact of weight on adverse health, particularly among older individuals. Reliance on selfreport of physician-diagnosed disease underestimates disease burden. The known association between overweight and chronic health conditions increases the likelihood of diagnosis in heavier people and represents an additional source of bias. In addition, using BMI as a weight measure provides an indirect measure of fatness and does not reflect fat distribution, which may affect the risk of co morbidity independent of BMI. Moreover, BMI does not distinguish between fat mass and lean tissue mass and may underestimate fatness in older adults who have greater amount of body fat at a given BMI than younger ones, due to age-related declines in muscle mass. Furthermore, we did not adjust our cross-sectional statistical models for variables often included in models that estimate incidence (rather than prevalence) of obesity-related co morbidities such as fat distribution, diet, or exercise, because the temporal sequence in crosssectional data is not established. Our results might have changed if these variables had been included. However, the consistency of our cross-sectional findings with incident chronic disease in several large prospective studies suggests that the cross-sectional associations we observed are likely to reflect true associations. The aforementioned misclassification and biases would likely have a minor impact on the magnitude of these estimates [5].

\section{Conclusion \& Recommendation}

Based on these results, $45 \%$ of overweight had hypertension, 36\% had DM, $9 \%$ had CVD \& 9\% had 


\section{Advances in Clinical Toxicology}

obesity. CVD \& 9\% had obesity. The prevalence of obesityrelated co morbidities emphasizes the need for concerted efforts to prevent and treat obesity rather than just its associated co morbidities. Overweight and obesity are common health conditions and their prevalence is increasing globally. Recent estimates suggest that 1 in 2 adults in the United States is overweight or obese, defined by a body mass index (BMI) of higher than 25, excess weight is associated with an increased incidence of cardiovascular disease, type 2 diabetes mellitus (DM), hypertension, stroke. Associations of excess weight with overweight- and obesity-related mortality may also have strong relation with personal habits mainly exercise.

In conclusion, development and implementation of obesity prevention strategies should target factors contributing to obesity, should target barriers to lifestyle change at personal, environmental and socioeconomic levels, and actively involve different levels of stakeholders and other major parties. We suggests that policy actions to the development and implementation of conditions. Effective public health strategies to obesity prevention should:

- Directly influence behavior, aiming at improving eating and physical activity behaviors.

- Target the food environments, the physical activity environments and the broader socioeconomic environments.

- Support health services and clinical interventions.

\section{References}

1. National heart lung and blood institution (2013), Why Obesity Is a Health Problem, Last Updated: February 13.

2. World health organization (2018) website, health topics-obesity.

3. Willett WC, Koplan JP, Nugent R, Dusenbury C, Puska $P$, et al. (2006) Disease Control Priorities in Developing Countries. $2^{\text {nd }} \quad$ (Edn.). Chapter 44Prevention of Chronic Disease by Means of Diet and Lifestyle Changes.

4. Park (2015) parks text books of preventive and social medicine 23rd (Edn.), Jabalpur, January, India.

5. By The MNT (Medical news today) (2016) Diabetes: Taking Insulin Editorial Team, Last updated Tue 5 January.

6. Cappuccio FP, Miller MA, Lockley SW, Rajaratnam SMW (2018) Sleep, Health and Society: from aetiology to public health. $2^{\text {nd }}($ Edn.), Oxford University Press.

7. Aviva M, Jennifer S, Eugenie H, Coakley MA, Colditz G, et al. (1999) The Disease Burden Associated with Overweight and Obesity. JAMA 282(16): 1523-1529.

8. SYSTEMS THINKING: OBESITY" (2016) The Annals of Pharmacotherapy: 39(12): 2046-2054. 